\title{
A Filter Algorithm for Suppressing Mixed Noise Based on Grey Relevance
}

\author{
SHEN De-hai ${ }^{a}, E X^{b}$, HOU Jian ${ }^{c}$, YAN Qi ${ }^{d}$
}

College of Information Science and Technology, Bohai University, Jinzhou, Liaoning 121013, China

ashendh25@qq.com, bexu21@163.com, ${ }^{c}$ jian_hou@163.com, d32392392@qq.com

Keywords: grey relevance, mixed noise, filter algorithm

Abstract. In order to suppress the mixed noise composed of salt \& pepper noise and Gauss noise in a digital image, a filter algorithm for suppressing mixed noise based on grey relevance is proposed. The algorithm classifies the noise point in the center of the filtering window, calculates the correlation coefficient of non-pepper and salt noise points in window by using the grey relational degree, and performs the weighted operation to replace the salt \& pepper noise point. For Gaussian noise point, the algorithm uses the mean of non-pepper and salt noise points in the filtering window to replace. The experiments results show that the algorithm can suppresses the mixed noise in images, and improves the sharpness of the image, which is better than traditional filtering algorithms.

\section{Introduction}

Noise will change the original pixel values in digital images, making the image feature is covered, which has great influence on the subsequent processing of image segmentation, tracking, information extraction and recognition. Therefore, suppressing image noise is an important prerequisite for image processing and analysis.

The traditional median filter and its improved algorithm [1-2] can effectively suppress salt \& pepper noise. Traditional mean filtering and its improved algorithm [3-4] can effectively suppress the Gaussian noise in a image, but actual images often contain both two kinds of noise, so the filtering method should take into account the mixed noise, and should retain the edge and other details in a image as much as possible while denoising.

Gray system theory was founded in 1982 by Chinese scholar Deng Julong [5]. It is a new method used to study the problem of uncertainty of information and data, and has been widely used in all fields of social sciences and natural sciences, and achieved good results. In the field of image processing, gray theory has also been applied to image compression, image hiding and image filtering, and achieved good results. Based on gray relational theory combined with the median and mean filtering algorithms, we propose a filtering algorithm suppressed mixed noise. The algorithm can suppress the salt \& pepper noise and the Gaussian noise mixed in a image perfectly, and has better protection for the edge details of the image and better visual effects.

\section{Grey relevance theory.}

Grey relevance analysis is one of the important concepts of grey system theory, and it mainly determines the degree of correlation between factors according to the internal relation and the similarity degree of development situation. The degree of relevance directly reflects the degree of influence of each factor in the system on the target value. The general steps of factor analysis using gray relational analysis are as follows:

1) Establishing the reference sequence and compared sequence.

According to the purpose of evaluation, we determine the evaluation index system, collect the data of system evaluation, and establish the reference sequence as shown in (1) and the comparison sequence as shown in (2). 


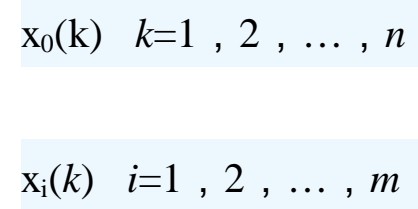

)

In Eq. 1 and Eq.2, $\boldsymbol{k}$ is the number of indicators, and $\boldsymbol{i}$ is the number of evaluation objects. The reference sequence should be an ideal comparison standard, which can be used as the reference data column with the optimal value (or the worst value) of each index, and other reference values can be selected according to the evaluation purpose.

2) Performing dimensionless processing on each series.

Data dimensionless is the main solution to the incomparability of data, and the commonly used dimensionless methods include means of mean, initial value and extreme value. Eq. 3 adopts the mean method.

$\mathrm{x}_{\mathrm{i}}(\mathrm{k})=\mathrm{x}_{\mathrm{i}}^{\prime}(k) / \frac{1}{\mathrm{~m}} \sum_{k=1}^{m} \mathrm{x}_{\mathrm{i}}^{\prime}(k)$

)

3) Calculating the correlation coefficient.

$\zeta_{i}(k)=\frac{\min _{i} \min _{k}\left|x_{0}(k)-x_{i}(k)\right|+\rho \max _{i} \max _{k}\left|x_{0}(k)-x_{i}(k)\right|}{\left|x_{0}(k)-x_{i}(k)\right|+\rho \max _{i} \max _{k}\left|x_{0}(k)-x_{i}(k)\right|} \quad i=1,2, \ldots n ; k=1,2, \ldots m$

)

In Eq.4, $\xi_{\mathrm{i}}(k)$ is correlation coefficient of each comparison sequence and reference sequence. $\left|x_{0}(k)-x_{i}(k)\right|$ is the absolute difference between the index sequence of each evaluated object (comparison sequence) and the reference sequence, $\min _{i} \min _{k}\left|x_{0}(k)-x_{i}(k)\right|$ and $\max _{i} \max _{k}\left|x_{0}(k)-x_{i}(k)\right|$ are the maximum and minimum values of these absolute values. $\rho$ is the value of the discrimination coefficient in $(0,1)$.

4) Solving the correlation degree.

The correlation coefficient of each index is weighted average by Eq.5, we can obtain the correlation degree of each evaluation object.

$$
r_{0 \mathrm{i}}^{\prime}=\frac{1}{\mathrm{~m}} \sum_{k=1}^{m} W_{k} \bullet \zeta_{\mathrm{i}}^{\prime}(k) \quad(k=1, \ldots m)
$$

\section{5 )}

\section{The proposed algorithm}

Because the features of these pixels contaminated by salt \& pepper noise and Gaussian noise are different, the uniform method cannot filter the noise well. The proposed algorithm filters the salt \& pepper noise and Gaussian noise respectively. For the salt \& pepper noise, the algorithm calculates the correlation coefficient by Carrying on the weighting operation to filter. For Gaussian noise, the algorithm uses the average value method to filter, reaching the better noise suppression effect, the concrete steps are as follows.

Noise detection. The algorithm uses $3 \times 3$ filtering window to detect noise. For each pixel in the image, if the pixel value is 0 or 255 , it is judged as the salt $\&$ pepper noise point, while others are considered as Gaussian noise points. The judgment method is shown as in Eq.6. 


$$
x_{i j \in}\left\{\begin{array}{lc}
\text { salt and pepper Noise } & x_{i j}=0 x_{i j}=255 \\
g \text { gaussian Noise } & \text { else }
\end{array}\right.
$$

Filtering salt \& pepper noise. If the center point of filter window is salt \& pepper noise, then the value of the noise point is replaced by the weighted value of each non-salt $\&$ pepper noise point by the gray relevance method. The algorithm is as follows.

1) Determining the comparison sequence and the reference sequence. The set of gray values of non-salt $\&$ pepper noise points in the filtering window are taken as the comparison sequence, suppose the set is $\boldsymbol{S}_{\mathrm{m}}, \boldsymbol{m}$ is the number of non-salt \& pepper noise points. The mean $\boldsymbol{s}$ of the value in set $\boldsymbol{S}_{\mathrm{m}}$ is computed as the value of the reference sequence.

2) Non-dimensional treatment. We use the average method in Eq.3 to perform non-dimensional treatment of comparison sequence and reference sequence.

3) Calculating the correlation coefficient. We use Eq.4 to calculate the correlation coefficient of each comparison sequence.

4) Calculating the weights. Using the normalization method to deal with each correlation coefficient, we get the result of $\boldsymbol{w}_{\mathrm{m}}$ as the weight of comparison sequence.

5) Weighting calculation. Each pixel value of non-salt \& pepper noise in the filtering window and its corresponding weights are weighted, the result replacements the noise point value in center of filtering window, the method is shown in Eq.7.

$$
\mathbf{x}_{i, j}^{\prime}=\sum_{s=1}^{m} w_{m} \bullet S_{m}
$$

Filter out Gaussian noise. If the center point of filtering window is Gaussian noise, in order to remove the effects of salt \& pepper noise, we use average of all the non-salt $\&$ pepper noise points in filtering window to replace the center pixel value.

\section{Verification experiment and the effect analysis}

Selects standard gray image lena with 256×256 pixels as initial image to make experiments, we mix different density mixed noise in initial image each time, use traditional median filtering algorithm (MedianF), traditional mean filtering algorithm (MeanF), and proposed algorithm(NewF) to filter noise image respectively, the size of filtering window of every algorithm is $3 \times 3$. The results are shown in Fig. 1 to Fig.3, the PSNR of these algorithms are shown in table1.

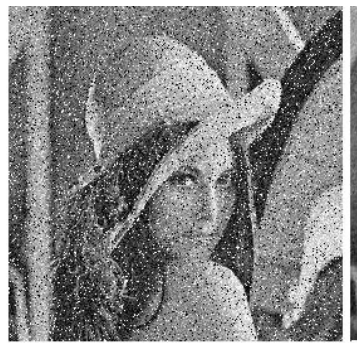

(a)

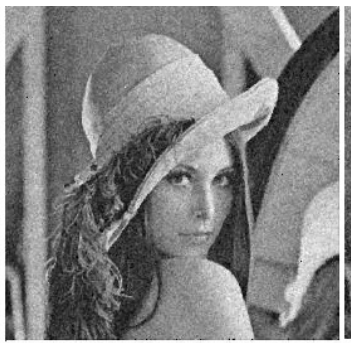

(b)

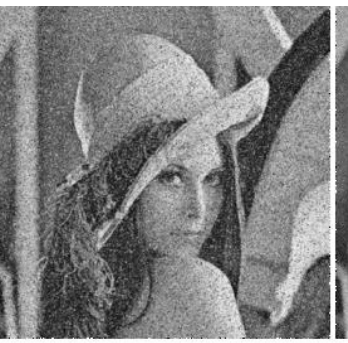

(c)

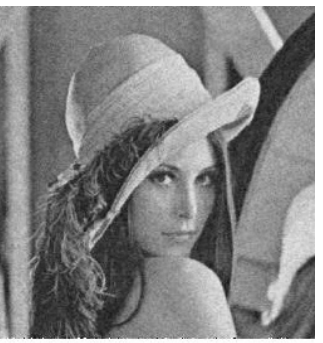

(d)

Fig 1 filtering image with low density Gauss noise and salt \& pepper noise 


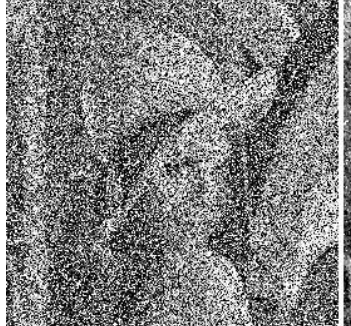

(a)

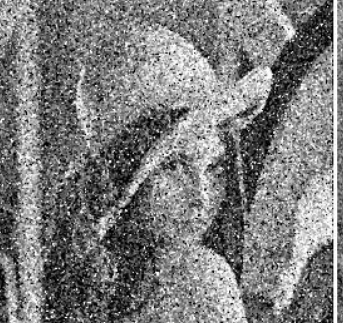

(b)

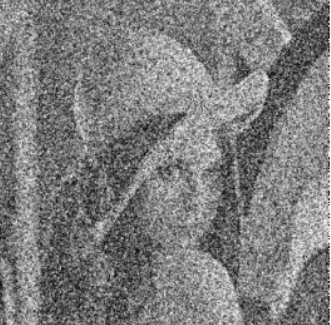

(c)

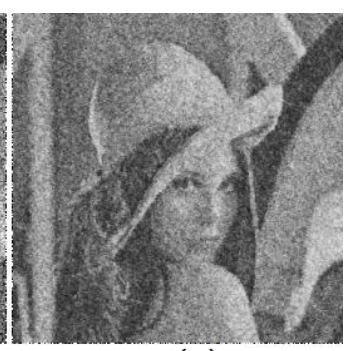

(d)

Fig 2 filtering image with middle density Gauss noise and salt $\&$ pepper noise

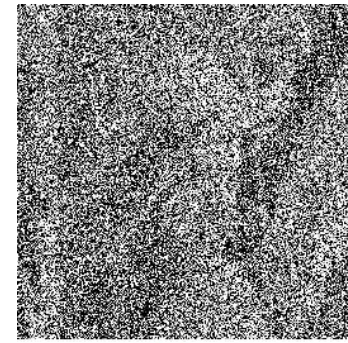

(a)

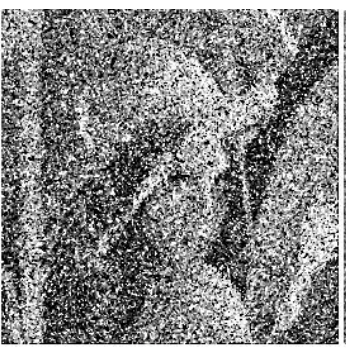

(b)

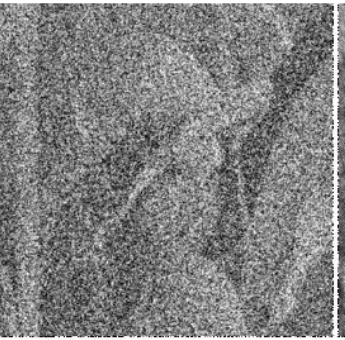

(c)

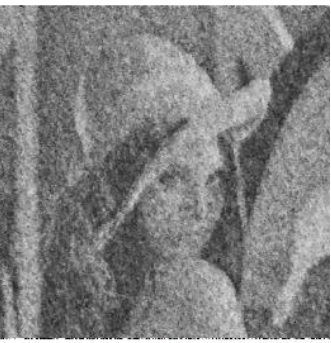

(d)

Fig 3 filtering image with high density Gauss noise and salt $\&$ pepper noise

In Fig.1-Fig.3, (a) is mixed noise image; (b) is MedianF filtering image; (c) is MeanF filtering image; (d) is proposed algorithm image.

In Fig.1, the initial image is mixed with Gauss noise of variance 0.01 (mean is 0 ) and salt \& pepper noise of 10\%. In Fig.2, the initial image is mixed with Gauss noise of variance 0.06 (mean is 0 ) and salt \& pepper noise of $40 \%$. In Fig.3, the initial image is mixed with Gauss noise of variance is 0.08 (mean is 0 ) and salt \& pepper noise of $60 \%$. It can be seen from the comparison images that the proposed algorithm $(\mathrm{NewF})$ has the best filtering performance under various noise concentrations. With the increase of the mixed noise concentration, the performance of the other two filtering algorithms decreases obviously, and the proposed algorithm can still filter out Most noise, the image edge is clearer than others.

Table 1 the PSNR comparisons of four algorithms

\begin{tabular}{lccccccc}
\hline Gauss noise & 0.01 & 0.04 & 0.05 & 0.06 & 0.07 & 0.08 & 0.10 \\
Salt \& pepper noise & $10 \%$ & $20 \%$ & $30 \%$ & $40 \%$ & $50 \%$ & $60 \%$ & $70 \%$ \\
\hline MedianF & 25.46 & 18.87 & 16.33 & 13.95 & 11.88 & 10.08 & 8.46 \\
MeanF & 22.71 & 19.03 & 17.40 & 16.18 & 15.21 & 14.36 & 13.55 \\
NewF & 27.21 & 22.24 & 21.08 & 20.28 & 19.45 & 18.81 & 17.86 \\
\hline
\end{tabular}

It can be seen from Table 1 that the PSNR values of the proposed algorithm are significantly higher than those of the two conventional filtering algorithms at different density noise disturbances, and the $P S N R$ value decreases slowly as the noise density increases.

\section{Conclusions}

In order to filter out the mixed noise, proposed a filter algorithm for suppressing mixed noise based on grey relevance. For salt \& pepper noise, the algorithm uses gray correlation method to calculate the weighting coefficient and uses weighted results to replace the noise point pixel. For the Gaussian noise, the algorithm uses average value of non-salt \& pepper noise in filtering window to replace the noise point pixel. Experiments show that the proposed algorithm has strong de-noising ability and image detail protection performance for different density mixed noise images, and the effect is obviously better than the traditional median filtering algorithm and the average filtering algorithm. 


\section{Acknowledgements}

This work was financially supported by the National Nature Science Foundation (61473045), Natural Science Foundation of Liaoning Province (20170540005), Science and Technology Research Projects of Department of Education of Liaoning Province (LQ2017003), Social Science Fund Projects of Liaoning Province (L17DJY002).

\section{References}

[1] Sun T, Neuvo Y. Detail-preserving median based filters in image processing[J]. Pattern Recognit Lett, 1994,15(4):341-347.

[2] Shen De-hai, LIU Da-cheng, XING Tao. A Multilevel Median Filter Algorithm Based On Vertical And Horizontal Windows Related. Computer Science, vol.39, no.5, p. 246-248, 2012, (In Chinese).

[3]Zhu Shihu, You Chunxia , A MODIFIED AVERAGE FILTERING ALGORITHM. Computer Applications and Software, vol.30, no.12, p. 97-99,116,2013, (In Chinese).

[4]Li Zuoyong, Tang KeZong, Hu Jinmei, et al. Directional weighted filter for image with salt \& pepper noise. Journal of Image and Graphics, vol.18,no.11,p.1407-1415, 2013(In Chinese).

[5]Deng Julong:Grey System Theory Tutorial (Huazhong University of Technology Press, China, 1992). 